\title{
Hallazgo de una hiperplasia lipomatosa del septum interauricular en un caso de muerte súbita
}

\section{Finding of a lipomatous hyperplasia in the interatrial septum in a case of sudden death}

\section{Resumen}

La hiperplasia lipomatosa del septum interauricular (HLSI) es una entidad benigna de naturaleza desconocida que se caracteriza por la acumulación de grasa no encapsulada en el interior del surco interauricular sin participación de la fosa oval. A pesar de su carácter benigno, clínicamente se ha asociado con arritmias cardiacas, generalmente de origen supraventricular, insuficiencia cardiaca y muerte súbita.

Presentamos el caso de una mujer de edad media (47 años), con obesidad mórbida e insuficiencia cardiaca congestiva, que falleció súbitamente y la autopsia puso de manifiesto una HLSI. El estudio macroscópico de la pieza de resección mostró un tamaño de $5 \times 2,5 \mathrm{~cm}$ y la histología típica de esta entidad, es decir, adipocitos maduros y pocos lipoblastos entremezclados con miocitos auriculares. Hemos analizado microscópicamente los nodos sinoauricular y auriculoventricular, lo que puso de manifiesto que la infiltración grasa los rodeada pero no los aislaba del miocardio auricular de trabajo circundante. El miocardio del ventrículo izquierdo y del tabique interventricular presentaba áreas con una intensa fibrosis intersticial por isquémica crónica y que esta fibrosis pudo ser la causa de una arritmia ventricular y muerte súbita.

Aunque esta entidad es cada vez más frecuentemente reconocida gracias al desarrollo creciente de las técnicas de imagen no invasivas, rara vez necesita ser corregida quirúrgicamente.

Palabras clave: Hiperplasia adiposa. Muerte súbita. Nodo sinoauricular. Nodo auriculoventricular. Septum auricular.

\section{Abstract}

The lipomatous hyperplasia of the interatrial septum (LHIS) is a benign entity of unknown nature, characterized by the accumulation of fat tissue not encapsulated into the interatrial groove without participation of the fossa ovalis. Despite its benign nature, it has been clinically associated with cardiac arrhythmias, usually of supraventricular origin, heart failure and sudden death.

We present the case of a woman of middle age (47 years), with morbid obesity and heart failure congestive who died suddenly and the autopsy revealed a LHIS. The macroscopic study of resection piece showed a size of $5 \times 2.5 \mathrm{~cm}$ and the typical histology of this entity, i.e. mature adipocytes and few lipoblastos interspersed with atrial myocytes. We have analysed microscopically both sinoatrial and atrioventricular nodes, which they revealed fat tissue infiltration that surrounded them but not isolated from atrial working myocardium. The interventricular septum and left ventricle myocardium presented areas with severe interstitial fibrosis by chronic ischemic and this fibrosis may be the cause of ventricular arrhythmia and sudden death.

Although this entity is most frequently recognized due to the development of noninvasive imaging techniques, rarely needs to be corrected surgically.

Key words: Adipose hyperplasia. Sudden death. Sinoatrial node. Atrioventricular node. Atrial septum.

\author{
D. Sánchez-Quintana ${ }^{1}$ \\ M. Murillo ${ }^{1}$ \\ F. Sánchez-Ugena² \\ S. Alama ${ }^{2}$ \\ MJ. Merino ${ }^{2}$ \\ MT. Sánchez ${ }^{2}$
}

${ }^{1}$ Departamento de Anatomía, Biología Celular y Zoología. Facultad de Medicina de Badajoz. Universidad de

Extremadura.

${ }^{2}$ Servicio de Patología Forense. Instituto de Medicina Legal de Badajoz.

Correspondencia: Dr. Félix Sánchez Ugena Servicio de Patología Forense.

IML de Badajoz.

Campus Universitario,

Parcela 5, s/n

06006 Badajoz

E-mail:

felix.sanchez@forense.mju.es

Fecha de recepción: 12.JUL.2011

Fecha de aceptación: 12.SEP.2011 


\section{Introducción}

La presencia de tejido graso en el corazón es un hallazgo frecuente en las autopsias, especialmente en personas de cierta edad ${ }^{1}$. La localización habitual del tejido adiposo es en el epicardio, infiltrando el miocardio (lipomatosis cardiaca) o por metaplasia grasa de un área miocárdica infartada.

Exponemos el caso de presencia de tejido adiposo de otra naturaleza. Se trata de la hiperplasia lipomatosa del septum interatrial (HLSI) que es un tumor benigno raro el cual se caracteriza por acumulación y depósito de grasa en el septum interatrial. Su etiología es aún desconocida, a pesar de que han sido sugeridas varias hipótesis. Normalmente se produce en personas de edad avanzada, obesas y con una mayor incidencia en el sexo femenino. En muchos casos permanece asintomática, siendo raro su diagnóstico en vida y siendo un hecho incidental durante la autopsia. En ocasiones la HLSI puede causar arritmias atriales tales como anormalidades de la onda$P$ y fibrilación atrial, otras veces obstrucción del flujo sanguíneo, y a veces muerte súbita ${ }^{1-3}$.

\section{Presentación del caso}

Mujer de 47 años de edad con obesidad mórbida, fumadora. Múltiples ingresos hospitalarios, el último de ellos tres meses antes de su muerte, por sobreinfección respiratoria. Antecedentes de fiebre reumática en su infancia. Diagnosticada de hipertensión arterial de larga evolución, miocardiopatía dilatada de posible origen enólico, insuficiencia aórtica moderada, insuficiencia cardiaca congestiva, EPOC y síndrome depresivo. Múltiples ingresos hospitalarios en relación con su síndrome de obesidad e hipoventilación asociada, el último de ellos tres meses antes de su muerte por clínica de insuficiencia cardiaca congestiva e insuficiencia respiratoria global reagudizada por sobre-infección respiratoria.

A primeras horas de la mañana del día de su fallecimiento, la familia refiere que presenta dificultad respiratoria y seguidamente pérdida de conciencia. Avisados los Servicios de Urgencia y tras aplicar maniobras de RCP avanzada, acreditan su defunción.

\section{Hallazgos de autopsia}

\section{Examen macroscópico}

Talla $163 \mathrm{~cm}$. Peso $150 \mathrm{Kg}$. Índice de masa corporal (IMC) $56,46 \mathrm{Kg} / \mathrm{m}^{2}$, lo que se corresponde con obesidad severa. Engrosamiento fibroso de la pleura pulmonar visceral (paquipleuritis), de mayor intensidad en el lado izquierdo. Pulmones aumentados de consistencia y de peso (D: 820 g. I: 840). En la superficie de sección del lóbulo superior del pulmón izquierdo, se aprecian focos confluyentes de aspecto bronconeumónico.

Peso del corazón 1.100 g (peso promedio estimado según peso corporal, 387 g; U95 de 587 g). Gran cantidad de grasa subepicárdica sin infiltración significativa del miocardio (Figura 1). Cardiomegalia intensa por insuficiencia aórtica (Figura 1) y mitral de probable etiología reumática. Válvulas pulmonar y tricúspide sin anomalías estructurales. Coronarias permeables. El espesor del miocardio en el ventrículo derecho a nivel de su ecuador es de $5 \mathrm{~mm}$, el del ventrículo izquierdo (VI) de $15 \mathrm{~mm}$, y el del septum interventricular de $2,5 \mathrm{~cm}$ (Figura 1 ). El VI está dilatado (unos 4,8 cm de diámetro) lo que junto con el peso elevado que posee son compatibles con el diagnóstico clínico de miocardiopatía dilatada (MCD) por lo que la cardiomegalia no es exclusiva de la insuficiencia aórtica. El miocardio presenta imágenes de pequeñas fibrosis o cicatrizaciones que afectan sobre todo a la pared diafragmática del ventrículo izquierdo (Figura 1). En el septum interatrial presenta una formación en cuña en el interior del surco interauricular posterior recubierta de epicardio y de aspecto lipomatoso de $5 \times 2,5 \mathrm{~cm}$. Macroscópicamente a la resección, la hiperplasia se presenta como un tumor adiposo no redondeado, circunscrito no encapsulado y de consistencia firme, que se extiende desde el techo de las aurículas a la parte anterior y posterior de la fosa oval, en el interior del surco interauricular posterior sin afectar a la fosa, pero interesando de forma obstructiva a las venas cavas, fundamentalmente a la inferior y a la vena pulmonar derecha superior (Figura 2).

\section{Estudio microscópico}

Histología de la masa extirpada. El estudio histológico revela que la formación grasa extirpada está constituida por una hiperplasia y acumulación de tejido adiposo (excepto en el área de la fosa oval) con ausencia de cápsula externa (Figura 3) que suele ser el epicardio o el endocardio. Lo más característico resulta la mezcla entre adipocitos maduros con citoplasma grande, claro y vacío, muy pocos de rasgos fetales, y haces musculares estriados compuestos por miocitos de tipo cardíaco (Figura 3), de tamaño anormalmente aumentado y de núcleos irregulares, rodeados en ocasiones por tejido conectivo. No hemos observado proliferación de estructuras vasculares o de elementos nerviosos en el interior o exterior de la masa adiposa. 
Método de estudio de los nodos cardiacos. El área de unión entre la vena cava superior con la aurícula derecha y su orejuela, así como el área de unión septal aurículoventricular (AV) a nivel del triángulo de Koch se extirparon en bloque del corazón constituyendo dos bloques de tejido. Los bloques posteriormente fueron deshidratados, incluidos en parafina y seccionados seriadamente a $10 \mu \mathrm{m}$ de espesor, según un eje sagital a la cresta terminal de la aurícula derecha para estudiar el nodo sinoauricular (Figura 4) o perpendicular al anillo tricuspídeo para estudiar el nodo auriculoventricular (Figura 5). De cada 10 secciones, 1 se montó y se tiñó con tricrómico de Masson.

Estudio histológico del miocardio ventricular. El miocardio del ventrículo izquierdo está parcialmente conservado y posee una intensa fibrosis miocárdica intersticial fundamentalmente subendocárdica (Figura 6) que reemplaza totalmente el miocardio que sufrió necrosis. También resulta llamativa el área de tejido conectivo denso cicatrizal que posee el septum interventricular (Figura 6). La fibrosis puede interpretarse como de origen isquémico secundaria a la importante hipertrofia ventricular ya que los troncos coronarios principales son permeables.

\section{Discusión}

El primer autor en describir en un estudio postmortem la hiperplasia lipomatosa del septum interauricular (HLSI) fue Prior en $1964^{4}$, y se caracteriza como hemos observado en nuestros hallazgos macro y microscópicos por una excesiva deposición de tejido graso en el interior del surco interauricular casi siempre mayor en su zona cefálica y anterior pero sin afectar a la fosa oval, por lo que el septum afecto adopta un aspecto de "campana" o "reloj de arena". El verdadero septum interauricular desde un punto de vista anatómico es la fosa oval ${ }^{5,6}$, por eso algunos autores, nosotros entre ellos, sugerimos otros nombres más que la
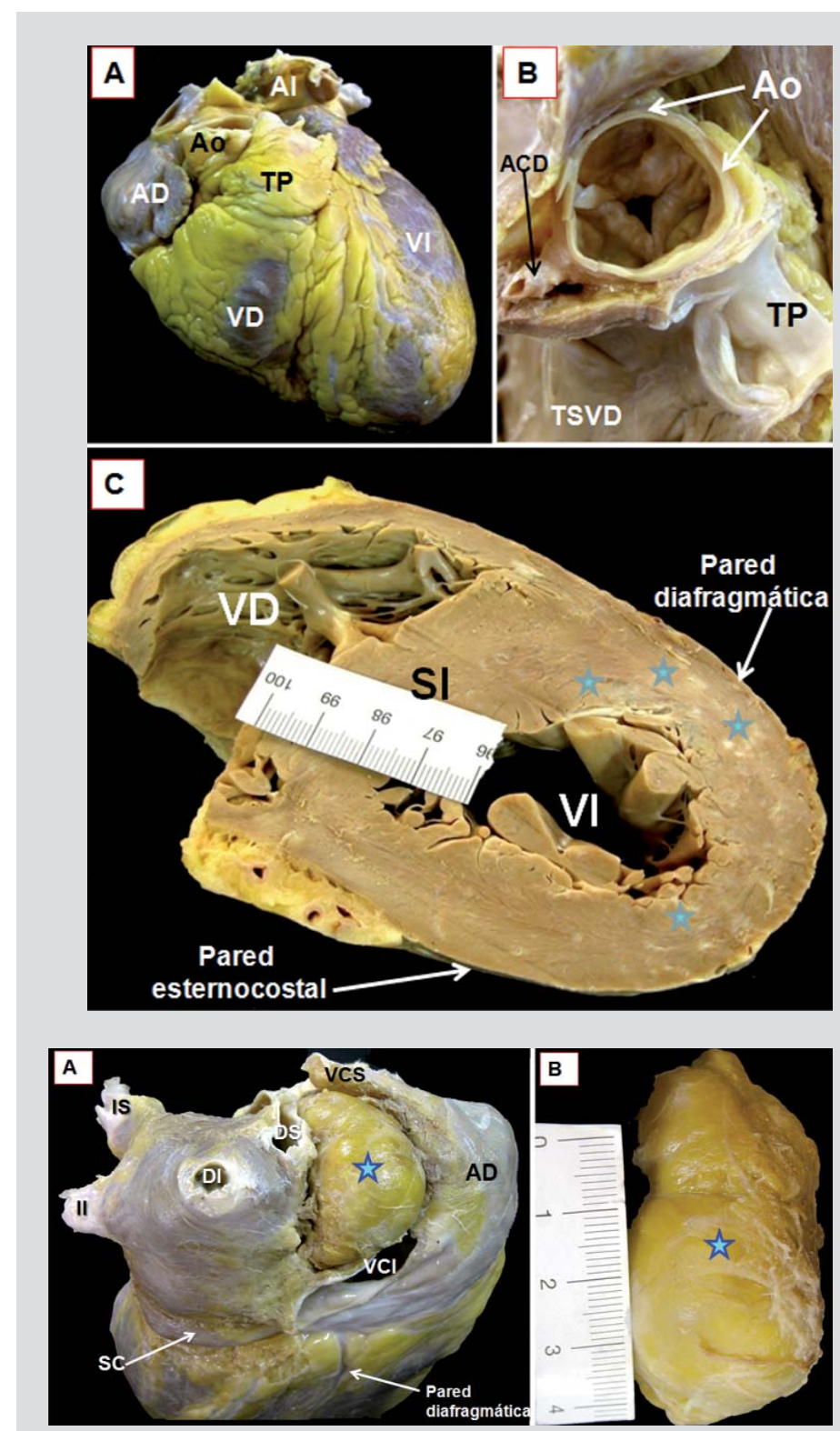

Figura 2.

A. Formación tumoral en el interior del surco interauricular posterior recubierta de epicardio (estrella). Nótese la compresión sobre las venas cavas y vena pulmonar derecha superior (DS) B. Tamaño macroscópico del tumor (estrella) una vez realizada su resección. VCS: Vena Cava Superior. VCl: Vena Cava inferior. $A D$ : Aurícula Derecha. DI: Vena Pulmonar Derecha Inferior. II: Vena Pulmonar Derecha Inferior. IS: Vena Pulmonar Izquierda Superior. SC: Seno Coronario.).

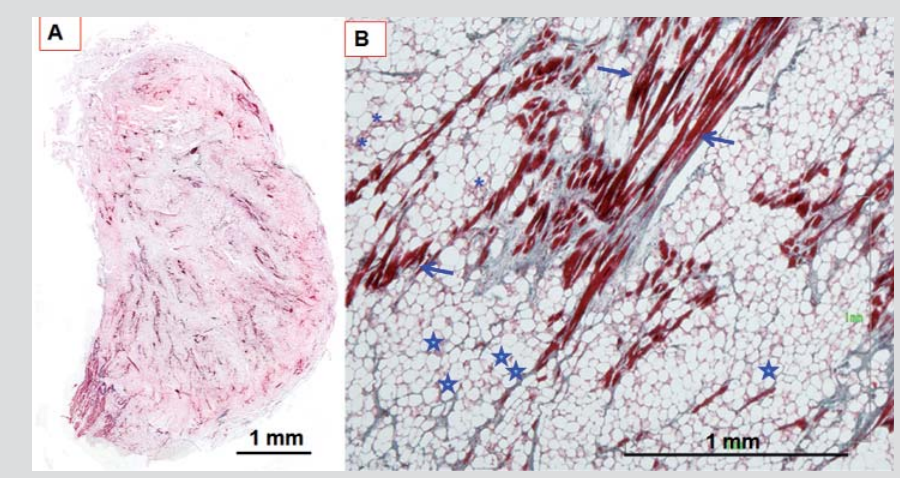

Figura 3.

A. Sección histológica horizontal del tumor graso teñida con tricrómico de Masson. Nótese como no es redondeado, es circunscrito y no encapsulado y no contiene en su interior vacuolas o líquido.

B. Sección histológica teñida con tricrómico de Masson en la que se observa la hiperplasia del tejido adiposo, mezcla entre adipocitos maduros con citoplasma grande (estrella), claro y vacío, algunos de rasgos fetales (asterisco), y miocitos de tipo cardíaco (fecha). 
denominación "septal", como "depósito masivo de grasa"7,8 en el interior del surco interauricular, "masa cardiaca y paracardiaca" 9 , "hamartoma lipomatoso"10. Tampoco está claro el concepto de "lipomatoso", que es incorrecto, debido a que los infiltrados grasos no son lipomas, pues los lipomas están encapsulados y no contienen grasa fetal ${ }^{11}$. Se le ha denominado también en vez de hiperplasia con la palabra "hipertrofia", lo cual es inapropiado porque la lesión es debido a una proliferación de células grasas o hiperplasia más que hipertrofia de los adipocitos $^{12}$. A pesar de todas estas incorrecciones, el término preferido por los autores, en las publicaciones más recientes, sigue siendo hipertrofia lipomatosa del septum interatrial.

No existe un criterio estricto que defina la HLSI aunque se considera anómalo el acúmulo de tejido adiposo entre un rango de menos de 1 a más de 2 $\mathrm{cm}^{1,8,13}$. Las células recuerdan al tejido adiposo pardo cuya función es principalmente energética generando calor durante el periodo postnatal precoz ${ }^{1}$. La prevalencia de la HLSI en pacientes vivos es de $2,8 \%{ }^{14}$, lo cual es mayor que los hallazgos necrópsicos que son solo del $1 \% 1,15$, aunque se estima que su frecuencia real es mayor ya que hasta el $8 \%$ de los ecocardiogramas muestran engrosamientos anómalos del septum interatrial $^{1,9}$. Las técnicas más apropiadas para su diagnóstico son la tomografía axial y la resonancia magnética ${ }^{12,16}$. En un estudio prospectivo reciente en 1.292 pacientes a los que se les había realizado tomografía axial por otras indicaciones, se observó HLSI en 29 pacientes $(2,2 \%)^{1}$. Desde que fue descrita en 1964 ${ }^{4}$, hasta el año 1992 se habían publicado 200 casos en todo el mundo ${ }^{17}$, hoy día se han publicado muchos más y su incidencia está relacionada con el sexo femenino, la obesidad y el incremento de la edad ${ }^{1}$, confirmando que la HLSI es más común de lo que previamente se sospechaba.

En general la HLSI suele ser detectada de manera casual y suele tener una clínica benigna en la mayoría de los pacientes, pero frecuentemente se asocia con arritmias auriculares como contracción auricular prematura, síndrome del seno enfermo ${ }^{2}$, flúter auricular ${ }^{3}$, y fibrilación auricular que con frecuencia requieren de fármacos antiarrítmicos ${ }^{1}$. En el caso que presentamos, por la historia clínica, la paciente no poseía trastornos arrítmicos auriculares, sin embargo, existe una ligera infiltración adiposa de los nodos, pero este hallazgo tiene en la paciente de nuestro caso una escasa repercusión funcional. El mecanismo de las arritmias auriculares en pacientes con HLSI es desconocido. Se ha sugerido que la incidencia de arritmias auriculares aumenta con el aumento del espesor de la grasa en el tabique interauricular ${ }^{18}$, lo que podría infiltrar de grasa el miocardio de la pared lateral auricular 
derecha y la cresta terminal, y esto puede interferir con la arquitectura y transmisión eléctrica entre los miocitos nodales y auriculares de trabajo, lo que produciría fenómenos de reentrada.

En cuanto a la causa de la muerte, en el caso que presentamos, el estudio muestra a una paciente que no poseía edad avanzada (47 años), los troncos coronarios principales estaban permeables y no se ha podido constatar la existencia de factores que relacionen la HLSI con la ateromatosis coronaria ${ }^{1}$. La histología del miocardio ventricular muestra que no existe infiltración adiposa, ni presencia de signos inflamatorios, ni atrofia. Lo más representativo es una intensa fibrosis subendocárdica, sobre todo a nivel del lado izquierdo del tabique interventricular, que puede interpretarse como de origen isquémico secundaria a la importante hipertrofia ventricular y que esta fibrosis intersticial pudo ser la causa de una arritmia ventricular y esta produjese la muerte súbita, con lo que descartamos la posibilidad de que la causa de la muerte fuera un foco infeccioso neumónico.

En la mayoría de los casos, la HLSI no requiere ningún tratamiento específico. El tratamiento quirúrgico

\section{Bibliografía}

1. Heyer CM, Kagel T, Lemburg SP, Bauer TT, Nicolas V. Lipomatous hypertrophy of the interatrial septum: a prospective study of incidence, imaging findings, and clinical symptoms. Chest 2003;124:2068-73.

2. Sato $Y$, Matsuo S, Kusama J, Kunimasa T, Yoda S, Matsumoto N, Tani S, Saito S. Lipomatous hypertrophy of the interatrial septum presenting as sick sinus syndrome. Int J Cardiol. 2007;119:280-1.

3. Dickerson JA, Smith M, Kalbfleisch S, Firstenberg MS. Lipomatous hypertrophy of the intraatrial septum resulting in right atrial inflow obstruction and atrial flutter. Ann Thorac Surg. 2010;89:1647-9.

4. Prior JT. Lipomatous hypertrophy of cardiac interatrial septum: a lesion resembling hibernoma, lipoblastomatosis and infiltrating lipoma. Arch $\mathrm{Pa}$ thol. 1964;78:11-5.

5. Anderson RH, Razavy R, Taylor AM. Cardiac anatomy revisited. J Anat. 2004;205:159-77.

6. Ho SY, Sánchez-Quintana D. The importance of atrial structure and fibers. Clinical Anatomy 2009;22:52-63. de HLSI no suele ser necesario ${ }^{19,20}$ y cuando se interviene quirúrgicamente debe limitarse a los pacientes con severos e intratables trastornos del ritmo, inestabilidad hemodinámica ${ }^{21}$, síntomas de síndrome de la vena cava superior o obstrucción de la aurícula derecha ${ }^{3,22-24}$. En nuestro caso, en la autopsia macroscópica observamos que la masa adiposa comprimía las venas cavas, sobre todo la inferior y la vena pulmonar derecha superior y la paciente presentaba manifestaciones clínicas de insuficiencia cardiaca congestiva y disfunción respiratoria.

\section{Conclusiones}

La hiperplasia lipomatosa del tabique interauricular se considera una patología cardiaca poco frecuente. Se trata de un tumor benigno formado por proliferación de tejido graso que infiltra el septum interatrial. A pesar de su carácter histológico de benignidad, puede ser causa de procesos arrítmicos que produzca la muerte súbita, de ahí su interés Médico Forense.
7. Shirani J, Roberts WC. Clinical, electrocardiographic and morphologic features of massive fatty deposits ("lipomatous hypertrophy") in the atrial septum. J Am Coll Cardiol. 1993;22:226-38.

8. Gay JD, Guileyardo JM, Townsend-Parchman JK, Ross K. Clinical and morphologic features of lipomatous hypertrophy ("massive fatty deposits") of the interatrial septum. Am J Forensic Med Pathol. 1996;18:107-8.

9. Wann LS, Sampson C, Liu Y. Cardiac and paracardiac masses: complementary role of echocardiography and magnetic resonance imaging. Echocardiography 1998;15:139-46.

10. Inoue T, Mohri N, Nagahara T, Takanashi R. A case report of "lipomatous hypertrophy of the cardiac interatrial septum", with a proposal for a new term "lipomatous hamartoma of the cardiac atrial septum". Acta Pathol Jpn. 1988;38:1583-9.

11. Isner JM, Swan CS, Mikus JP, Carter BL. Lipomatous hypertrophy of the interatrial septum: in vivo diagnosis. Circulation 1982;66:470-3.

12. O'Connor S, Recavarren R, Nichols LC, Parwani AV. Lipomatous hypertrophy of the interatrial septum: an overview. Arch Path Lab Med. 2006;130:397-9. 
13. Meaney JF, Kazerooni EA, Jamadar DA, et al. CT appearance of lipomatous hypertrophy of the interatrial septum. Am J Roentgenol. 1997; 168:1081-4.

14. Kuester LB, Fischman AJ, Chieh-Min Fan C-M, Halpern EF, Aquino SL. Lipomatous hypertrophy of the interatrial septum. Prevalence and features on fusion $18 \mathrm{~F}$ Fluorodeoxyglucose Positron Emission Tomography/CT. Chest 2005; 128;3888-93.

15. Reyes CV, Jablokow VR. Lipomatous hypertrophy of the cardiac interatrial septum: a report of 38 cases and review of the literature. Am J Clin Pathol. 1979;72:785-8.

16. Stephant E, Barthelet M, Leroux PY, Revel D. Images in cardiovascular medicine. Lipomatous hypertrophy of the interventricular septum: echocardiography, cardiac magnetic resonance, and multidetector computerized tomography imaging. Circulation 2008;118:e71-72.

17. Pochis WT, Saeian K, Sagar KB. Usefulness of transesophageal echocardiography in diagnosing lipomatous hypertrophy of the atrial septum with comparison to transthorasic echocardiography. $\mathrm{Am}$ J Cardiol. 1992;70:396-8.

18. Aziz YF, Julsrud PR. Can cardiac magnetic resonance imaging reliably differentiate between benign and neoplastic fat? Int J Cardiovasc Imaging 2002;18:227-30.
19. Nadra I, Dawson D, Schmitz SA, Punjabi PP, Nihoyannopoulos P. Lipomatous hypertrophy of the interatrial septum: a commonly misdiagnosed mass often leading to unnecessary cardiac surgery. Heart 2004;90:e66-e69.

20. Koniari I, Koletti B, Apostolakis E. Lipomatous hypertrophy of the atrial septum: clinical approach and surgical indications. Interact CardioVasc Thorac Surg. 2011;12:512-3.

21. Calé R, Andrade MJ, Canada M, Hernandez-Estefania R, Lima S, Abecasis M, Vitorino E, Gouveia R, Gouveia R, Silva JA. Lipomatous hypertrophy of the interatrial septum: report of two cases where histological examination and surgical intervention were unavoidable. Eur J Echocardiogr. 2009; 10:876-9.

22. Breuer M, Wippermann J, Franke U, Wahlers T. Lipomatous hypertrophy of the interatrial septum and upper right atrial inflow obstruction. Eur J Cardiothorac Surg. 2002;22:1023-5.

23. Scholten KJ, Soran PD, Van der Starre PJA. Obstruction of pulmonary artery catheterization because of lipomatous hypertrophy of the interatrial septum. J Cardiothorac Vasc Anesth. 2008;22:751-2.

24. Garmendia M, Rodríguez I, Ereño C, Bilbao FJ, López JI. Hipertrofia adiposa del septo interauricular. Presentación de un caso quirúrgico. Rev Esp Patol. 2008;41:142-5

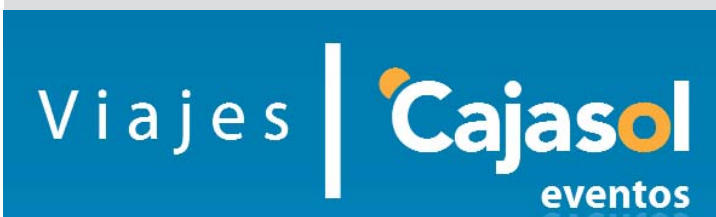

Contamos con un Departamento dedicado exclusivamente a la Organización Profesional de todo tipo de Eventos: Congresos, Simposios, Eventos Deportivos/Culturales, Reuniones/Encuentros, Cursos/Jornadas, etc.

Con los mejores y más modernos equipos de trabajo, personal altamente cualificado y experiencia demostrable. Avanzadas Tecnologías de Gestión de Congresos, con equipos informáticos específicos para la organización de eventos.

Reconocido prestigio empresarial, por planificación, viabilidad económica, gestión exclusiva, y disponibilidad territorial con logística para diseñar eventos en cualquier punto de España o Resto del Mundo.

Para nosotros, cada evento tiene que salir perfecto Contáctenos y compruébelo

www.viajescajasol.es

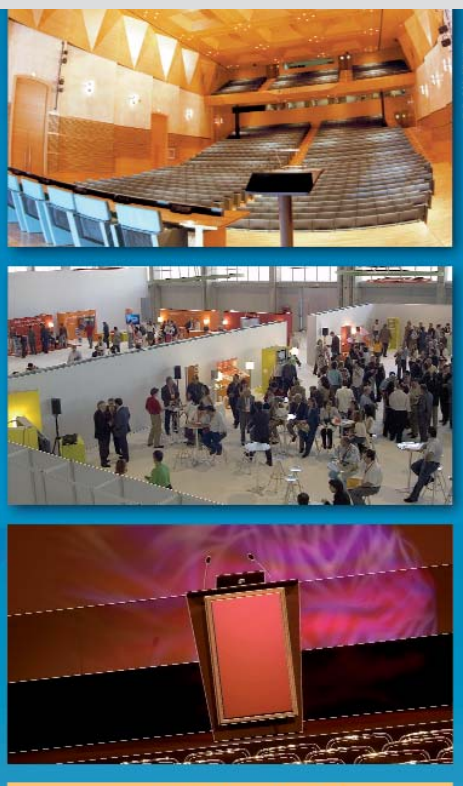

Oficina de Eventos: Santo Domingo de la Calzada, 5 41018 Sevilla 954981089 eventos@viajescajasol.es 